\title{
Caracterización e identificación morfométrica de las ubres de cabras criollas (Capra aegagrus hircus) de la parroquia Colonche, ubicados en el bosque deciduo de tierras bajas de Ecuador
}

\author{
Acosta Lozano Néstor Vicente \\ ORCIDhttps://orcid.org/0000-0003-4848-1360 \\ nacosta@upse.edu.ec \\ Universidad Estatal Península de Santa Elena \\ Santa Elena, Ecuador
}

\author{
Chávez García Debbie Shirley \\ ORCID: https://orcid.org/0000-0001-8157-2761 \\ dchavez@upse.edu.ec \\ Universidad Estatal Península de Santa Elena \\ Santa Elena, Ecuador
}

\author{
González De La A María Isabel \\ ORCID:https://orcid.org/0000-0001-6035-1409 \\ mariagonza1447@gmail.com \\ Universidad Estatal Península de Santa Elena \\ Santa Elena, Ecuador
}

Recibido (23/04/21), Aceptado (14/05/21)

\begin{abstract}
Resumen: El objetivo fue caracterizar la morfología de las ubres de cabras criollas de Colonche, se evaluaron 73 hembras adultas, las variables fueron, tamaño y ángulo de pezones, profundidad, inserción y conformación general de ubre. Se estableció un análisis estadístico descriptivo para las variables fanerópticas y morfométrica con el software estadístico SPSS versión 20. Los resultados determinaron que las cabras criollas tienen caracteres mamarios con una ubre periforme con un tamaño de pezón $5,23 \mathrm{~cm}$, ideal para la producción de leche, un ángulo de inserción fuerte y amplio $5,93 \mathrm{~cm}$, con buena profundidad de ubre $4,84 \mathrm{~cm}$ un ángulo de los pezones direccionados verticalmente $6,21 \mathrm{~cm}$ y una conformación general ideal en casi todas hembras. Las medidas morfológicas de la glándula mamaria de la ubre fueron: profundidad $17,52 \mathrm{~cm}$, perímetro $23,59 \mathrm{~cm}$, distancia entre pezones $9,12 \mathrm{~cm}$, surco intermamario $4,35 \mathrm{~cm}$, longitud del pezón $2,60 \mathrm{~cm}$, diámetro base del pezón $2,7 \mathrm{~cm}$, predominan animales de capa policromada, de pelo corto.
\end{abstract}

Palabras Clave: Cabras criollas, cisterna, glándula mamaria, pezón.

\section{Characterization and morphometric identification of the udders of Creole goats (Capra aegagrus hircus) from the Colonche parish, located in the lowland deciduous forest of Ecuador}

\begin{abstract}
The objective was to characterize the morphology of the udders of Creole goats of Colonche, 73 adult females were evaluated, the variables were, size and angle of teats, depth, insertion and general udder conformation. A descriptive statistical analysis was established for the phaneroptic and morphometric variables with the statistical software SPSS version 20. The results determined that the Creole goats have mammary characters with a pear-shaped udder with a $5.23 \mathrm{~cm}$ nipple size, ideal for milk production. a strong and wide insertion angle $5.93 \mathrm{~cm}$, with good udder depth $4.84 \mathrm{~cm}$ a vertically directed teat angle $6.21 \mathrm{~cm}$ and an ideal general conformation in almost all females. The morphological measurements of the mammary gland of the udder were: depth $17.52 \mathrm{~cm}$, perimeter $23.59 \mathrm{~cm}$, distance between teats $9.12 \mathrm{~cm}$, intermammary groove $4.35 \mathrm{~cm}$, length of the nipple $2.60 \mathrm{~cm}$, base diameter of the nipple $2.7 \mathrm{~cm}$, animals with a polychrome coat and short hair predominate.
\end{abstract}

Keywords: Creole goats, cistern, mammary gland, nipple.

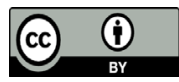




\section{Introducción}

La glándula mamaria es una sección de los animales (hembras) misma que tiene como función principal producir y ofrecer al recién nacido un fácil acceso a la leche materna, se encuentra suspendida por afuera de la pared del abdomen posterior y no se encuentra unida, soportada o protegida por estructura ósea [1].

Las cabras en su mayoría presentan glándulas mamarias abultadas cuando son multíparas y en el caso de las jóvenes estas se encuentran recogidas, pero aumentan y cambian de características de acuerdo al número de partos y lactancias que estas hayan presentado a lo largo de su vida reproductiva. Además, estas se encuentran condicionadas de acuerdo a la forma y tamaño de la ubre, donde intervienen factores tales como la raza y las condiciones ambientales, las cuales

determinan la fabricación o no de la producción de la leche [2].

El origen de las glándulas mamarias indica nacer desde la sección inguinal, entre las extremidades posteriores; está conformada por dos bolsas, separados por un ligamento y cubiertas por un tejido glandular denominados conductos, donde es transportada la leche hacia el exterior a través de la extracción y la acción de la alimentación de la cría o el ordeño [3] [1]. La leche es almacenada en la parte inferior de la glándula conocido como cisterna y es expulsada a través del pezón [4]. Se estima que, para producir un litro de leche, es necesario que llegue a la ubre 425 litros de sangre [5].

Su forma varía según la edad del animal, siendo más globosa en animales adultos y multíparas, presentando un colgamiento a medida que aumenta el número de partos y la longitud de los pezones es de 3 a $5 \mathrm{~cm}$, están ubicados de forma lateral y dirigidos cranealmente, una de las características es que las cabras desarrollan pezones extra-numerarios [6].

La morfología y fisiología de la glándula mamaria tiene su importancia en la clasificación del ganado caprino dentro de los sistemas de producción de leche, en los tiempos actuales existen ensayos en caracteres mamarios en razas caprinas lecheras [7]. En la evaluación de caracteres morfológicos existen métodos de evaluación en la glándula mamaria, el cual consiste en la aplicación de técnicas de valorización lineal, análogos al del ganado bovino lechero [8].

Dentro del método de valoración estándar en la evaluación de las glándulas y de una escala lineal que parte desde el rango más bajo hasta el más alto (1-9 puntos), donde las valoraciones con categoría 5 corresponden al valor intermedio [5]. Existen factores de riesgo para la evaluación de la ubre asociados a la morfología del pezón, relacionados con la forma, el tamaño y diámetro del pezón, lo cual ha generado datos que sugieren condiciones para el establecimiento de diversos padecimientos, estos pueden ser por ejemplo la distancia de los pezones al suelo, que facilita el comienzo de infección intramamaria; también la forma del pezón y de la punta del mismo, ya que pezones largos y gruesos se han asociados a un mayor riesgo para la salud de la ubre, aunque solo la longitud del pezón resultaría independiente de la incidencia de mastitis ([9], [10],[11]).
El sistema mamario es considerado por muchos autores como una de las aptitudes determinantes para los sistemas productivos de los rumiantes menores en producción de leche [12].

Con lo antes expuesto el trabajo de investigación planteo como objetivo caracterizar la morfología de las ubres de cabras criollas que se localizaron en la parroquia Colonche ubicado en el bosque deciduo de tierras bajas de Ecuador y se evaluó el tamaño y ángulo de pezones, profundidad, inserción y conformación general de ubre para determinar sus especificaciones.

\section{Desarrollo}

La existencia de grupos de especies animales con riqueza genética adaptados y reconocidos por su capacidad de producir y reproducir bajo condiciones poco favorables y en estrés, están constituidas con base a grupos genéticos de razas criollas [11], con criterios que permiten su integración a sistemas de producción específicos y la ventaja de resistencia de estos grupos genéticos a diversos factores de estrés y enfermedades [13]. Por consiguiente, se recomienda incluir el empleo de las características morfológicas del pezón en programas de mejoramiento genético, por la heredabilidad de media a alta que esta presenta en sus crías [3].

El destino de la leche caprina producida en los traspatios principalmente es de autoconsumo de la familia y en escasas ocasiones para la comercialización [11].

La posibilidad de seleccionar las reproductoras de acuerdo con las características de la morfología del pezón y ubres en ganadería tropical, podría ser una estrategia útil para acompañar el proceso de modernización del sistema de producción [11].

\section{Metodología}

La presente investigación se realizó en la parroquia Colonche, cantón y provincia de Santa Elena, Ecuador que presenta una ubicación de -2.01667 de latitud Sur y -80.667 de longitud Oeste cuenta con una extensión de 1137,2 km2, el clima que presenta es seco, con una temperatura promedio anual de $25^{\circ} \mathrm{C}$, precipitación promedio de $328 \mathrm{~mm}$ anuales y una altitud de $655 \mathrm{msnm}$ [14].

Para realizar el trabajo de investigación se utilizaron 73 cabras criollas adultas únicamente que estén gestando y paridas como factor importante para la caracterización de las glándulas mamarias. Para la determinación de los caracteres morfológicos de la ubre se utilizó la metodología descrita por Vanegas en la clasificación de tipología de la ubre periforme (A), oval (B), globosa (C) como lo muestra (Figura 1). Estas medidas fueron valoradas de forma lineal y con parámetros establecidos en la literatura. Las variables en estudio fueron, tamaño y ángulo de los pezones, profundidad, inserción y conformación general de la ubre y la tipología [1]. 


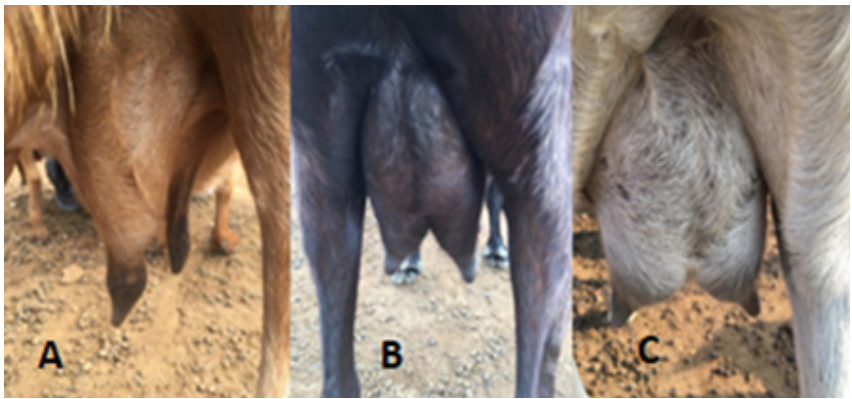

Figura 1. Tipología de la ubre

En cuanto a las medidas morfométricas Figura 2, se tomaron en consideración variables como perímetro de inserción (A), profundidad (B), perímetro (C) de la ubre, distancia entre pezones (D), altura de cisterna (E), surco intermamario $(\mathrm{F})$, variables de estudio que se consideraron en el pezón $(\mathrm{G})$; longitud, diámetro y posición del pezón.

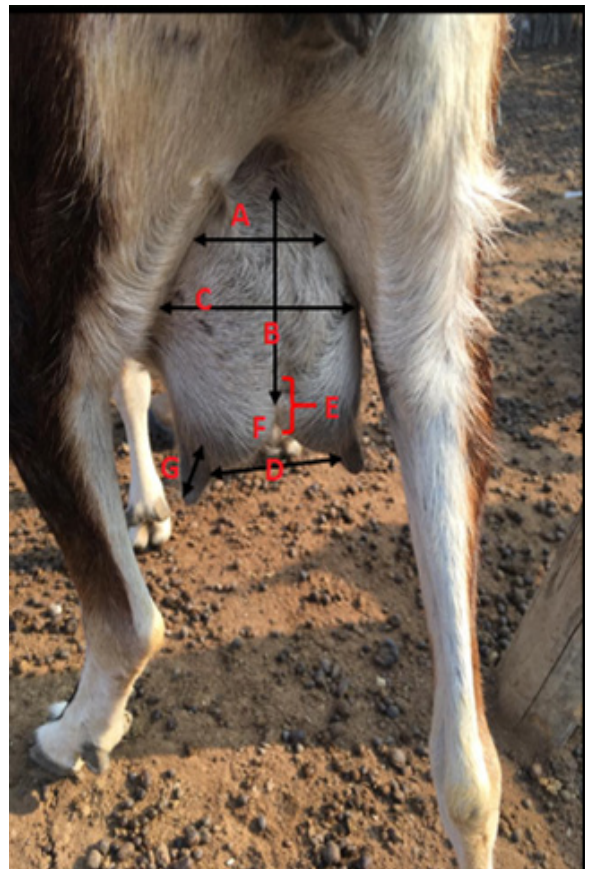

Figura 2. Medidas morfométricas de las ubres

Para la caracterización morfométrica y faneróptica de las cabras criollas existentes en el área de estudio se utilizaron las variables alzada de la cruz y la grupa, ancho de oreja, cabeza y grupa, diámetro bicostal, longitud del cuello, cabeza, oreja, grupa y cuerpo, altura del corvejón, perímetro de caña y torax, peso vivo, ancho de cadera, diámetro dorso esternal, color de las capas de pelo, perfil frontonasal, forma de la mordida, tamaño y tipo de pelo y cuerno, presencia o ausencia de mamelas, dirección de orejas. Para las medidas morfométricas en estudio de las ubres se utilizaron como instrumentos de medida el pie de rey vernier, bastón y cintas zoométricas.

En la colecta de la información se usó una hoja de cálculo de Excel y en el análisis de datos se aplicó un diseño estadís- tico descriptivo en las variables fanerópticas y morfométricas apoyados en el paquete estadístico SPSS versión 20.

\section{IV.Resultados}

Dentro de los principales resultados se puede observar en la Tabla 1 las características fanerópticas de las glándulas mamaria de 73 cabras criollas evaluadas en la parroquia Colonche y se observó en la forma de la ubre un $42,5 \%$ de forma oval o descolgada, periforme $38,4 \%$ y globular $19,2 \%$, estos resultados discrepan con los reportados en el ensayo realizado en cabras criollas del cantón Zapotillo de la provincia de Loja quienes encontraron el tipo de ubre predominante en esa zona son la forma oval o descolgada con un $41,53 \%$, las ubres globosas y descolgadas obtuvieron un menor proporción de $3,6 \%$ [15]. Se manifiesta que las ubres más apreciadas para la reproducción son las que se aproximan a una semiesfera y bien fijadas en el abdomen, estudios realizados en Valencia, España indican que las características en un rebaño de ovejas Guirras con una tipología I (descolgada) son mejores productoras de leche [5].

De acuerdo con los caracteres lineales se situaron en la profundidad media de la ubre aquellas que no sobrepasan los corvejones siendo estas las características adecuado para esta variable presentando un total de $41,1 \%$ de las cabras mientras que $27,4 \%$ tienen ubres que sobrepasan el corvejón teniendo una distancia muy corta al suelo y $31,5 \%$ ubres muy altas por encima de los corvejones. La profundidad de la ubre presenta una condición de 5 puntos que equivale a una media que se aproxima a lo ideal dentro de un juzgamiento de la especie [16]. Mientras que el perímetro de inserción que es considerado como de sujeción del pezón de la glándula mamaria solo $34,2 \%$ de las ubres de las cabras en estudio fueron consideradas fuertes y amplias, $54,8 \%$ medios y $11 \%$ débiles, mientras más fuertes es su inserción menos será la tendencia a descolgarse. Para la verticalidad de los pezones lo que se define como el ángulo de posición o implantación del pezón lo que facilita el ordeño el 39,7\% tienen un ángulo de implantación vertical que son los aconsejables para el sistema productivo de leche, el $60,3 \%$ son de características pocos deseables.

El tamaño de los pezones deber ser apropiado para garantizar el fácil ordeño sea manual o mecánico esto se refiere que los pezones intermedios son los más apropiados así se evidencio que 47 animales que representan el 64,4\% tienen el tamaño ideal, 15 animales presentan pezones grandes que son el $20,5 \%$ y un $15,1 \%$ son pequeños, y respecto a la conformación de la ubre esta también correlacionada con el ordeño, encontrándose que 53,4\% tienen una conformación intermedia y un $37 \%$ están en una conformación deseable.

Indicando el valor promedio de "inserción de la ubre" de 5 puntos (deseables), y un promedio de verticalidad de 6 puntos (5-6 normales) con tamaño de pezón de 4 puntos, un punto inferior al de este estudio que están dentro de los tamaños normales y la conformación de la ubre con puntuaciones de 5 alejándose de la condición adecuada [17]. 
Tabla 1. Distribución de frecuencia faneróptica de las glándulas mamarias de la cabra criolla de la parroquia Colonche

\begin{tabular}{l|ccccc}
\hline Variables & Categorías & Frecuencia & $\begin{array}{c}\text { Porcentaje } \\
\mathbf{( \% )}\end{array}$ & $\begin{array}{c}\text { Porcentaje } \\
\text { válido (\%) }\end{array}$ & $\begin{array}{c}\text { Porcentaje } \\
\text { acumulado } \\
\text { (\%) }\end{array}$ \\
\hline \multirow{2}{*}{ Forma de la ubre } & Periforme & 28 & 38,4 & 38,4 & 38,4 \\
& Oval & 31 & 42,5 & 42,5 & 80,8 \\
\hline Medidas lineales & & 14 & 19,2 & 19,2 & 100 \\
\hline \multirow{2}{*}{ Profundidad de la } & PP & 23 & 31,5 & 31,5 & 31,5 \\
ubre & PM & 30 & 41,1 & 41,1 & 72,6 \\
\hline \multirow{2}{*}{ Perímetro de } & MP & 20 & 27,4 & 27,4 & 100 \\
\hline inserción de la ubre & ID & 8 & 11 & 11 & 11 \\
\hline \multirow{2}{*}{ Ángulos de los } & IM & 40 & 54,8 & 54,8 & 65,8 \\
pezones & IFA & 25 & 34,2 & 34,2 & 100 \\
\hline \multirow{2}{*}{ Tamaño de los } & IH & 7 & 9,6 & 9,6 & 9,6 \\
pezones & IL & 37 & 50,7 & 50,7 & 60,3 \\
\hline \multirow{2}{*}{$\begin{array}{l}\text { Conformación de la } \\
\text { ubre }\end{array}$} & IV & 29 & 39,7 & 39,7 & 100 \\
\hline & PP & 11 & 15,1 & 15,1 & 28,8 \\
\hline & PI & 47 & 64,4 & 64,4 & 79,5 \\
& CD & 15 & 20,5 & 20,5 & 100 \\
\hline
\end{tabular}

PP: poco profunda, PM: profundas medias, MP: muy profundas, ID: inserción débil, IM: inserción mediana, IO: inserción fuerte y amplia, IH: implantación horizontal, IL: Implantación lateral, IV: Implantación vertical, PP: pezones pequeños, PI: pezones intermedios, PG: Pezones grandes, CN: Conformación no apropiada, CI: conformación intermedia, CD: conformación deseable.

En las características morfológicas de la ubre presento que la profundidad corresponde a la distancia tomada verticalmente desde la inserción perineal y el ligamento suspensor medio de la base de la ubre por lo que los animales en estudio obtuvieron una media de $17,52 \mathrm{~cm}$, con un perímetro de inserción que mide la inserción de la pared abdominal teniendo una media de $20,67 \mathrm{~cm}$, estos valores discrepan con los reportados por Hernández donde indican una profundidad y perímetro de la ubre $16,90 \mathrm{~cm}, 34 \mathrm{~cm}$ respectivamente, promedio de distancias y longitud de pezones de $13,98 \mathrm{~cm}$, $2,07 \mathrm{~cm}$ [19]. El perímetro de la ubre se midió en la distancia máxima que esta dio cuyo valor fue $23,59 \mathrm{~cm}$, la distancia entre pezones se midió desde los puntos de inserción interna de cada uno de los pezones y presento un valor de 9,12 $\mathrm{cm}$, mientras que el surco intermamario indico una medida de $2,68 \mathrm{~cm}$ tomadas entre las dos mitades de la ubre y la profundidad del surco, la longitud del pezón fue tomada desde la base del pezón y el extremo de este reportando una medida de 2,68 cm, el diámetro de la base del pezón reporto $2,67 \mathrm{~cm}$ y la altura de cisterna $2,4 \mathrm{~cm}$ medida que se otorgó desde el punto de inserción del pezón y la parte ventral de la cisterna.

En estudios similares realizados en Venezuela y Veracruz, las cabras obtuvieron una profundidad de ubre que varía entre 11 a $24 \mathrm{~cm}$ y un perímetro de $23,50 \mathrm{~cm}$ [18] siendo estos valores similares a los encontrados en esta investigación debido a la similitud de las condiciones ambientales; sin embargo, respecto a la distancia de pezones en esa misma investigación difiere debido que encontraron pezones de mayor tamaño con $12,5 \mathrm{~cm}$, y en la longitud de pezones de $6,64 \mathrm{~cm}$ [18]. 
Tabla 2. Caracterización morfométrica de las glándulas mamarias de la cabra criolla de la parroquia Colonche.

\begin{tabular}{l|cccccc}
\hline \multicolumn{1}{c|}{ Variables (cm) } & N & Mínimo & Máximo & Media & Desv. típ. & Varianza \\
\hline Profundidad de la ubre & 73 & 5 & 28 & 17,52 & 7,002 & 49,031 \\
Perímetro de inserción & 73 & 4 & 36 & 20,67 & 8,780 & 77,085 \\
Perímetro de la ubre & 73 & 3 & 40 & 23,59 & 8,787 & 77,218 \\
Distancia entre pezones & 73 & 2 & 19 & 9,12 & 3,460 & 11,971 \\
Surco intramamario & 73 & 1 & 10 & 4,34 & 2,070 & 4,284 \\
Longitud del pezón & 73 & 1 & 6 & 2,68 & 1,066 & 1,135 \\
Diámetro base del pezón & 73 & 1 & 6 & 2,67 & 1,248 & 1,557 \\
Altura de la cisterna & 73 & 0 & 4 & 2,40 & 1,164 & 1,354 \\
\hline
\end{tabular}

En la ganadería caprina criolla existe una diversidad de colores debido a los diferentes cruces que se realizan en los hatos donde se practica la reproducción de cruces cerrados, adicional estas están relacionadas con el tipo de clima, la altitud y el tipo de suelo en que ellas se desarrollan, en la variable de variedades de colores que se encontraron al realizar el estudio, determinado que el color de capa con mayor frecuencia es el Overo representado en $21,9 \%$, seguido del negro con patas de color bayas $19,2 \%$ y la tonalidad tostada de $13,7 \%$ de igual forma el color con menos predominancia fue el negro y blanco. En lo que respecta al tipo de pelaje se reporta un $91,8 \%$ de pelo el lizo y $47,9 \%$ con un tamaño de pelaje mediano, $46,6 \%$ pelo corto y en menor frecuencia el pelaje crespo y tamaño largo con 8,2 y $5,5 \%$ respectivamente, esto se presenta en cabras localizadas en regiones cálidas y despoblados predominando el color negro con café, ya que poseen una mayor tolerancia al sol a diferencia de las cabras de tonalidad blanca y colores claros, esta característica se ven reflejadas en los resultados obtenidos apreciando menor frecuencias en las cabras de color blancas coincidiendo con trabajos realizados sobre sistemas caprinos del norte de la provincia de Córdoba [20], sin embargo son similares a los presentados en la caracterización de la cabra del cantón Zapotillo, Loja al mencionar que las cabras registran un tipo de pelaje liso mientras que en pocos animales se registraron animales con un pelaje crespo, el $99 \%$ de la población con un pelaje corto pegado al cuerpo y solo $1 \%$ pelaje largo [15],[8].

Las cabras en el estudio presentaron un perfil frontonasal recto siendo la mayor proporción representado por un 67,1 $\%$, mientras que $32,9 \%$ poseen un perfil cóncavo, en lo que respecta a la mordida los resultados muestran que dentro de los parámetros normales se representan a $82,2 \%$ y $16,45 \%$ presentan prognatismo, las cabras presentaron cuernos arqueados en un $64,4 \%$ y en forma de espiral $23,3 \%$ y con menos frecuencia animales sin cuernos o denominados mochos con $12,3 \%$; en cuanto a la presencia de mamelas un 76,7 $\%$ de ellas no las tienen y $23,3 \%$ presentaron ausencia, con respecto a la dirección de las orejas $74 \%$ de ellas son caídas, $19,2 \%$ horizontales y un $6,8 \%$ erguidas siendo estas menos frecuentes dentro del grupo de estudio.

La caracterización de cabras de esta región andina presenta un $60 \%$ animales con perfil un frontonasal recto, mientras que el $40 \%$ presentaba un perfil cóncavo, todos estos valores están correlacionadas con los resultados obtenidos en el estudio, el $66 \%$ presentaron cuernos en forma de arco y el $2 \%$ cuernos en forma de Prisca (espiral), el 7,2 \% de la población caprina demostraron ser animales sin presencia de cuernos (mochos) [17]. La mayoría de la población caprina en la parroquia Colonche en Santa Elena no presentaron mamelas, esta característica coincide con estudios realizados en Zapotillo donde $85 \%$ de los animales no presentaron mamellas con orejas caídas y de tamaño grande [8].

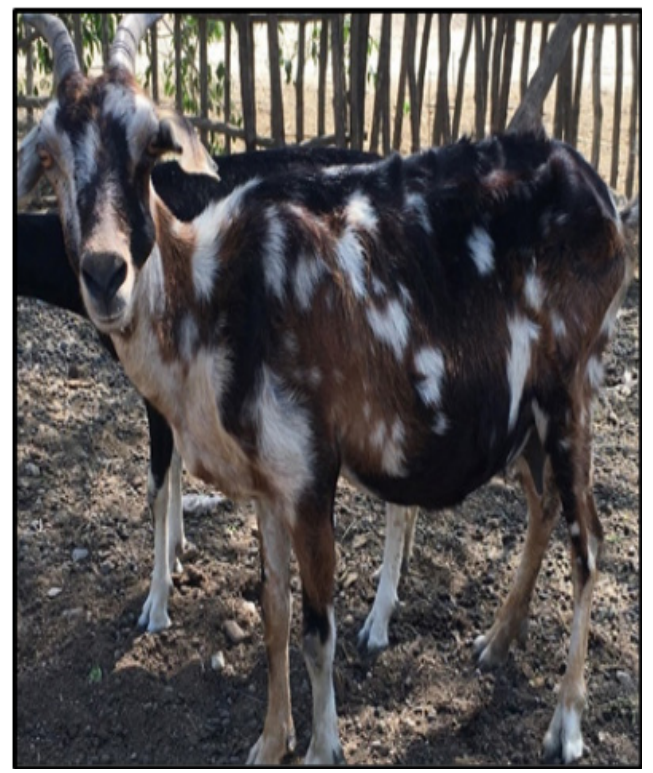

Figura 3. Características fenotípicas principales de caprino criollo localizado en la parroquia Colonche

En la Tabla 3 se observa las variables zoométricas, indicando el promedio de alzada a la cruz de $68,1 \pm 6,3 \mathrm{~cm}$ y una 
altura de la grupa de $65,7 \pm 7,7 \mathrm{~cm}$ estas medidas se encuentran dentro del rango reportado por ensayos realizados en la Loja uno de los sectores de mayor presencia de población caprina, e indica que la alzada de la cruz fue de $64,5-68,3 \mathrm{~cm}$ en hembras adultas conociendo que esta característica se ve influenciada del genotipo Anglo Nubia y las cabras hembras llegan a tener un ancho de grupa de $16 \mathrm{~cm}$ [15], [22]; con un ancho de oreja, cabeza y grupa de $6,96 \pm 0,9 \mathrm{~cm}, 20,49 \pm 2,68$ $\mathrm{cm}, 15,22 \pm 2,18 \mathrm{~cm}$ respectivamente, un diámetro bicostal promedio 44,82 $\pm 5,02 \mathrm{~cm}$, longitud del cuello, cabeza, corporal y oreja obtuvieron $20,86 \pm 3,48,23,86 \pm 3,47,62,81 \pm$ $7,26,16,7 \pm 2,10 \mathrm{~cm}$ cada una de sus medidas. Con una altura del corvejón de $18,22 \pm 3,06 \mathrm{~cm}$, perímetros de la caña y toraxico $7,93 \pm 1,122 \mathrm{~cm}, 71,37 \pm 6,89 \mathrm{~cm}$, longitud de la grupa $14,96 \pm 2,26 \mathrm{~cm}$, un ancho de cadera y diámetro dorso esternal de $17,92 \pm 2,89 ; 58,47 \pm 9,19 \mathrm{~cm}$ y peso vivo promedio de las cabras evaluadas $26,21 \pm 6,4 \mathrm{~kg}$ estos valores se discrepan de otras investigaciones donde se determinaron pesos vivos de $22,4 \mathrm{~kg}$ en las hembras adultas [8], [22]. Otros autores reportan un ancho de oreja de $7 \pm 0,5 \mathrm{~cm}$, mostrando ser unas orejas de características corta y angosta y un ancho de cabeza $20,89 \mathrm{~cm}$ para machos y las hembras mostraron un valor de $18,20 \mathrm{~cm}$ en etapa de adultez, a la vez reportaron un ancho de cabeza de 14,90 cm, una longitud corporal entre $65-71 \mathrm{~cm}$ en etapa adulta, perímetro torácico de $85,7 \mathrm{~cm}$ y longitud de la grupa promedio $23 \mathrm{~cm}$ [17] siendo estos valores similares a los encontrados en la presente investigación, otros ensayos manifiestan que las cabras obtuvieron un promedio de la alzada de la cruz 55,9 y $72,40 \mathrm{~cm}$, con un diámetro biscotal de $18,5 \mathrm{~cm}$, longitud de orejas de $5,60 \mathrm{~cm}$, longitud de cuello de $19 \mathrm{~cm}$, longitud de cabeza de 16,20 - 21,70 cm [23], [24], [25] coincidiendo con este estudio realizado.

Tabla 3. Caracterización zoométricas de la cabra criolla de la parroquia Colonche

\begin{tabular}{l|cccccc}
\hline Variables & N & Mínimo & Máximo & Media & Desv. típ. & Varianza \\
\hline \hline ACR (cm) & 73 & 53 & 95 & 68,10 & 6,329 & 40,060 \\
AGR (cm) & 73 & 30 & 94 & 65,70 & 7,693 & 59,186 \\
AO (cm) & 73 & 5 & 10 & 6,96 & 0,949 & 0,901 \\
ACZ (cm) & 73 & 12 & 25 & 20,49 & 2,688 & 7,226 \\
AGR (cm) & 73 & 11 & 23 & 15,22 & 2,181 & 4,757 \\
DB (cm) & 73 & 30 & 59 & 44,82 & 5,023 & 25,232 \\
LC (cm) & 73 & 13 & 29 & 20,86 & 3,477 & 12,092 \\
LCZ (cm) & 73 & 16 & 31 & 23,86 & 2,978 & 8,870 \\
LCU (cm) & 73 & 46 & 75 & 62,81 & 7,264 & 52,768 \\
ACO (cm) & 73 & 7 & 25 & 18,22 & 3,006 & 9,035 \\
LO (cm) & 73 & 12 & 21 & 16,70 & 2,100 & 4,408 \\
PC (cm) & 73 & 6 & 12 & 7,93 & 1,122 & 1,259 \\
PT (cm) & 73 & 57 & 86 & 71,37 & 6,689 & 44,736 \\
LG (cm) & 73 & 10 & 22 & 14,96 & 2,257 & 5,096 \\
PV (kg) & 73 & 15 & 45 & 26,21 & 6,401 & 40,971 \\
ACD (cm) & 73 & 11 & 29 & 17,92 & 2,895 & 8,382 \\
DDE (cm) & 73 & 30 & 77 & 58,47 & 9,189 & 84,447 \\
\hline \hline
\end{tabular}

ACR: Alzada a la cruz , AGR: Alzada a la grupa , AO: Ancho de oreja, ACZ: Ancho de cabeza, AGR: Ancho de grupa, DB: Diámetro bicostal, LC: Longitud del cuello, LCA: Longitud de Cabeza, LCU: Longitud del Cuerpo, ACO: Altura del corvejón, LO: Longitud de oreja, PC: Perímetro de caña, PT: Perímetro torácico, LG: Longitud de grupa, PV: Peso vivo, ACD: Ancho de cadera, DDE: Diámetro dorso esternal 
Estas características son las que identifican a las cabras criollas encontradas en la parroquia Colonche provincia de Santa Elena y son el resultado de cruzamientos no controlados e introducción de otras razas procedentes principalmente de Loja que está ubicada al sur del país existiendo la mayor producción caprina de Ecuador [21].

\section{Conclusiones}

Las cabras de biotipo criolla localizadas en la parroquia Colonche presentan caracteres mamarios ideales para la producción de leche presentando una ubre de tipología periforme o alargada con un tamaño de pezón ideal con un determinado ángulo de inserción fuerte y amplio y una buena profundidad de la ubre, un ángulo de los pezones direccionados verticalmente y una conformación general ideal lo que es favorable el ordeño de las cabras.

Dentro de la morfológica de la glándula mamaria se determinó una buena profundidad de la ubre con características de perímetro de la ubre, distancia entre pezones, surco intermamario, longitud del pezón, diámetro de la base del pezón características óptimos para producción de leche.

Las características fenotípicas de los caprinos en la zona de estudio indica que predominan los animales de capa policromada (varios colores), de pelo corto y liso, orejas grandes y caídas, tienen cuernos en forma de arco y un perfil frontonasal recto y sin mamelas lo que ha favorecido en las condiciones medio ambientales en las que se desarrollan expuesto a los diferentes sistemas de manejo.

\section{Referencias}

[1]B.Vanegas García, "Caracterización de la morfología mamaria y control lechero en un rebaño comercial de ovejas Guirras", Valencia: Universitad Politécnica de Valéncia, 2013.

[2]V. Landi, "Conservación de los recursos zoogenticos", Panama, 2011. [Online]

Available at: http://www.uco.es/conbiand/pdf/libro_resumenes_panama2012.pdf

[3]M. Riera-Nieves, V. Vila-Vals and E. Perozo-Prieto, "Características morfológicas de los pezones y su relación con la producción de leche y eficiencia de ordeño en vacas de raza Carora" Revista Científica FCV - LUZ. vol 18, n 6, pp 734 $-738,2008$.

[4]L. Rodríguez, A. Hernández, A. Cervantes, M. Domínguez and J.Martínez, "Morfometría de los pezones de vacas doble propósito en hatos de la zona ganadera de Veracruz", trabajo presentado en la Reunión Científica Tecnológica Forestal y Agropecuaria Veracruz, Colombia, 2013.

[5]J. Vázquez García, "Relación de medidas de la glándula mamaria de cabras raza nubia en la producción y calidad de la leche", 2013.[ Online]

Available at: http://ninive.uaslp.mx/xmlui/handle/i/3426

[Último acceso: 2603 2020].

[6]K. González, "La reproducción de las cabras”, 2017 [Online]

Available at: https://zoovetesmipasion.com/cabras/la-repro- duccion-de-las-cabras/

[Último acceso: 2002 2020].

[7]G. Ferrano, "Lactación de la cabra y los factores que la regulan", 2010. [Online]

Available at: https://helvia.uco.es/xmlui/bitstream/handle/10396/3770/02-1990

[8]A. Flores, "Caracterización fenotípica de la cabra criolla y su sistema de producción, en la Parroquia Limones del Cantón Zapotillo", 2018. [Online] Available at: https://dspace.unl.edu.ec/jspui/bitstream/123456789/20535/1/alex\%20 maximiliano\%20flores\%20gonz\%c3\%81lez.pdf.

[9]R. Singh, B. Bansal and D.Gupta, "Udder health in relation to udder and teat morphometry in Holstein Friesian× Sahiwal crossbred dairy cows", Tropical animal health and production, vol. 46, pp. 93-98, 2014.

[10]T. Buragohain, P. Das, P. Ghosh, D. Banerjee and J. Mukherjee, "Association between udder morphology and in vitro activity of milk leukocytes in high yielding crossbred cows", Vet World, vol.10, pp. 342-347, 2017.

[11]A. Hernández, P. Cervantes, B. Domínguez, M. Barrientos and F. Gómez, "Morfología del pezón en vacas de lechería en el trópico de Veracruz, México", Actas Iberoamericanas en Conservación Animal AICA vol. 10, pp. 251-256, 2017.

[12]N. Fernández , “Aptitud al ordeño mecánico de ovejas de raza Manchega: II. Producción de leche, reparto de fracciones y cinética de emisión de leche", In: 3rd International Symposium on Machine Milking of Small Ruminants. ed. Valladolid, Spain: Sever-Cuesta, 1983.XXXX

[13]A. Rojas, "Selección de cabras lecheras", 2001. [Online] Available at: http://biblioteca.inia.cl/medios/biblioteca/ta/ NR27087.pdf.

[14]Chávez, D., Villacrés Matías, J. "Estimación del gasto energético de los caprinos en la Península de Santa Elena", Revista Científica y Tecnológica UPSE. Vol 5, no. 1, pp. 7076.2018

[15]V. Villavicencio , "Caracterización morfológica de la cabra criolla del Ecuador en el Cantón Zapotillo, provincia de Loja", 2015. [Online]. Available: http://dspace.espoch. edu.ec/bitstream/123456789/5331/1/Tesis\%20Victor\%20Javier\%20Arias\%20Villavicencio.pdf..

[16]N. C. Gómez Urviola, “Caracterización estructural, morfológica y genética de la población de cabras autóctonas de la región apurímac del Perú", 2013. [Online]. Available: https:// hdl.handle.net/10803/125720.

[17]O. Camacho, "Caracterización fenotípica de la cabra criolla y su sistema de producción, en la parroquia Mangahurco del cantón Zapotillo", 2018. [Online]. Available: https://dspace.unl.edu.ec/jspui/bitstream/123456789/20971/1/ osvaldo \%20vladimiro $\% 20$ camacho $\% 20$ enriquez.pdf.

[18]G. Contreras, Z. Chirinos, S. Zambrano, E. Molero, "Caracterización morfológica e índices zoométricos de vacas Criollo Limonero de Venezuela", Rev. Fac. Agron, vol. 28, no. 1, pp. 91-103, 2011.

[19]C. Hernández, "Caracterización morfométrica de la cabra en el centro de Veracruz", 2015. [Online]. Available: http://132.248.9.34/hevila/Agroproductividad/2015/vol8/ 
no6/10.pdf?fbclid=IwAR1IkGSsKuDzmd9_MX9V_zbjiaZG-.

[20]M. Arias y A. Alonso, "Estudios sobre sistemas caprinos del norte de la provincia de Córdoba, Argentina", Archivos de Zootecnia, vol. 51, pp. 195, 2002.

[21]A. Solis Lucas, M. Lanari y M.Oyarzaba, "Phenotupic characterization of the goat population of Santa Elena province (Ecuador)", Archivos de Zootecnia, vol. 265, no 265, pp. 22-29, 2020.

[22]C. Landacay Barrera, "Caracterización fenotípica del ganado caprino criollo en el cantón Catamayo, provincia de Loja”, 2014. [Online]. Available: https://dspace.unl.edu.ec/ jspui/handle/123456789/12373.

[23]W. O. Burgos Paz, R. E. Vásquez Romero, J. O. Pérez Palencia, D. H. Bejarano Garavito y E. R. Camargo Sánchez, "La cabra criolla santandereana, patrimonio genético de Colombia”, 2015. [Online]. Available: http://repository.agrosavia.co/handle/20.500.12324/11551

[24]S. Carné, N. Roig y J. Jordana, "La cabra blanca de Rasquera: caracterización morfológia y faneróptica", Archivos de zootecnia, vol. 56, no. 215, pp. 319-330, 2007.

[25]Suárez, V.H.; Martínez, G.M.; Nievas, J.D.; Quiroga, Roger. J., "Prácticas de manejo y producción en sistemas familiares de cría caprina en las quebradas áridas de Jujuy y Salta RIA”, Revista de Investigaciones Agropecuarias, vol. 43, no. 2, pp. 186 -194, agosto, 2017.

\section{RESUMEN CURRICULAR}

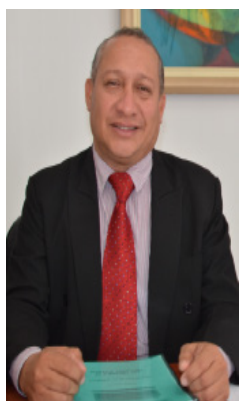

Acosta-Lozano N. Ingeniero Zootecnista, Master en producción Animal con mención en Nutrición y Alimentación Animal, Doctor en Ciencias Veterinarias, actual Vicerrector Académico de la Universidad Estatal Península de Santa Elena, participa en REDES de colaboración con varias universidades ha escrito varios artículos científicos de alto impacto en Scopus, Scielo y Latindex.

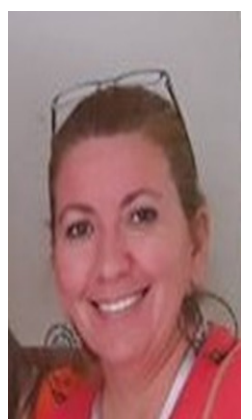

Chávez-García D. Médico Veterinario Zootecnista Magister en Clínica y Cirugía, Docente-Investigador UPSE, Director de proyectos con fines de conservación de animales autóctonos de la Península de Santa Elena, participa en REDES de colaboración con varias universidades, autora de diversas publicaciones en revistas indexadas a Scielo y Latindex, ha partic ipado en eventos nacionales e internacionales

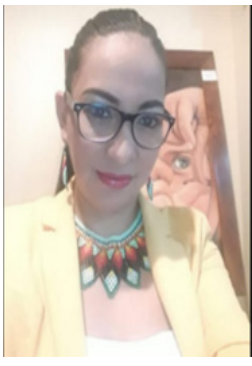

Andrade-Yucailla V. Ing. Zootecnista, maestría en Salud Animal Avanza y Doctora en Ciencias Veterinarias, Docente/Investigador de la UPSE, participa en proyectos de investigación nacionales e internacionales de diferentes IES como Investigadora y directora, participa en REDES con varias universidades ecuatoriana y extranjeras, participa en eventos científicos nacionales e internacionales y ha escrito varios artículos científicos de alto impacto en Scopus y Latindex.

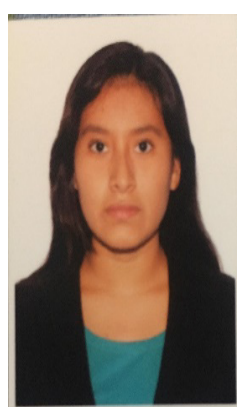

Gonzales de la A M. Estudiante de la Facultad de Ciencias Agraria, Carrera de Ingeniería Agropecuaria de la Universidad Estatal Península de Santa Elena. Participa en el proyecto de investigación sobre conservación de animales autóctonos del Instituto de Investigación Científica y Desarrollo de Tecnologías UPSE. 